\title{
PARTERAS TRADICIONALES Y PARTO MEDICALIZADO, ¿̇UN CONFLICTO DEL PASADO? EVOLUCIÓN DEL DISCURSO DE LOS ORGANISMOS INTERNACIONALES EN LOS ÚlTIMOS VEINTE AÑOS
}

\author{
Traditional Birth Attendants and Medicalized Birth, a Conflict From the Past? \\ Evolution of the Discourse of International Health Organizations in the Past Twenty Years \\ Hilda E. Argüello-Avendaño \\ Ana Mateo-González
}

Resumen: Este artículo se centra en el actual conflicto sobre el papel que deben desempeñar las parteras tradicionales en la asistencia a la reproducción —embarazo, parto y puerperio - y los paralelismos con el momento en que la partería dejó de ser un oficio de mujeres. Los discursos de organismos internacionales que dictan las políticas públicas sobre salud materna - UNFPA, OMS — nos recuerdan lo sucedido siglos antes en el momento en que la práctica médica se consolidaba como profesión y ciencia universal y oficial. A través de éstos se expresa el cuestionamiento sobre las capacidades de las parteras, en primer lugar para atender complicaciones y, posteriormente, para atender partos normales —eutócicos-, lo que coloca a las parteras como figuras externas al sistema sanitario oficial.

Palabras clave: parteras tradicionales, personal de salud calificado, mortalidad materna, atención materna.

Abstract: This article focuses on the current conflict over the role of traditional birth attendants in the assistance to the reproduction (pregnancy, childbirth and puerperium) and the similarities between this and the time in which midwifery was not longer more a women's job. The speeches of international agencies that dictate the public politics on maternal health - UNFPA, WHO — remind us what happened centuries ago in Europe and the United States, at the time that medical practice was consolidated as a profession and universal and official science. Through these questioning about the capabilities of midwives, first expressed to address complications and then for normal deliveries —eutocic - placing them as outside the formal health system figures.

Keywords: traditional birth attendant, skilled health worker, skilled attendant, maternal mortality, maternal care.

Hilda E. Argüello Avendaño, doctorante en antropología por la Universitat Rovira i Virgili. Investigadora asociada y asistente del secretariado técnico del Observatorio de Mortalidad Materna en México. Temas de especialización: salud materna, mortalidad materna, utilización de servicios de salud en contextos multiculturales, relación médico-paciente, violencia familiar, evaluación de política pública. Correo electrónico: hildaeu@gmail.com.

Ana Mateo González, doctorante en antropología de la medicina por la Universitat Rovira i Virgili. Desk de Gestión de Calidad en Médicos del Mundo, España. Temas de especialización: cooperación, inclusión social, pertinencia cultural, salud sexual y reproductiva. Correo electrónico: anamaria.mateo@medicosdelmundo.org.
Enviado a dictamen: 22 de enero de 2014. Aprobación: 21 de marzo de 2014.

Revisiones: 1 . 


\section{Introducción}

E ámbito de la asistencia a la reproducción embarazo, parto y puerperio- por parte de la llamada medicina occidental o biomedicina es relativamente reciente debido a que anteriormente el parto no era considerado un evento patológico y se relegaba a un plano "natural" y de mujeres. La "comadre" o "partera" era la encargada de asistir y ayudar a parir. Es en el siglo XVIII cuando la hegemonía de las parteras se pone sistemáticamente en cuestión desde el poder político, intelectual y religioso, y es sustituida de forma progresiva por la de los cirujanos comadrones. Esta transición, de la partería femenina no profesionalizada a la obstetricia primordialmente masculina, es un proceso inconcluso y complejo vigente aún en nuestros días.

Sin negar el derecho de las mujeres a una atención especializada cuando se presentan complicaciones —emergencias obstétricas-, la partería parece ir más allá de la atención obstétrica de la biomedicina ya que no se circunscribe a la atención del parto. Va más allá pues parte de la comprensión de lo que todo el proceso del embarazo/parto/puerperio significa dentro de la sociedad en la que se desenvuelve. Desde la década de los noventa se vive una transformación en el discurso de los organismos internacionales con respecto al papel de las parteras tradicionales en la asistencia a la reproducción. En los años setenta se optó por su formación para mejorar los servicios obstétricos allá donde no hubiera personal sanitario profesional. La Declaración de Alma Ata de 1978 sugiere la formación y utilización de los recursos humanos necesarios para el desarrollo de la estrategia primaria — que debía estar orientada a los principales problemas de salud, como la morbi-mortalidad materna e infantil-y la necesidad de proporcionar servicios básicos de salud. Hoy en día esta estrategia se encuentra desprestigiada y cobra fuerza la tendencia a posicionar a las parteras tradicionales como figuras externas al sistema sanitario. En este sentido se dan contradicciones entre el discurso - de los organismos internacionales-y la práctica, sobre todo en los países o regiones más empobrecidos donde los ministerios de salud continúan con la estrategia de capacitar parteras tradicionales como recurso de salud y, paradójicamente, en muchos casos con financiamiento de estos mismos organismos.

El discurso que se va configurando muestra similitudes a lo sucedido siglos antes en Europa y Estados Unidos, en el momento en que la práctica médica se consolidaba como profesión y ciencia universal y oficial, cuya legitimación requería estudios universitarios y licencia para ser ejercida. Lo que pretendemos es mostrar el actual conflicto entre el papel de las parteras tradicionales y el sistema sanitario oficial en la asistencia a la reproducción: mujeres en su mayoría, indígenas, pobres, rurales, que se enfrentan a una especialidad médica primordialmente masculina y occidental. Para ello mostraremos las inconsistencias entre el discurso ${ }^{1}$ y la práctica de la todavía baja atención del parto por parte de personal calificado o cualificado a nivel mundial. Continuaremos con un breve repaso del proceso de medicalización del parto, ${ }^{2}$ para concluir con el análisis de la transformación del discurso desde principios de los noventa de los Organismos internacionales ${ }^{3}$ que dictan las normas referentes a la salud sexual y reproductiva.

\section{El papel de la partera en la historia}

El nacimiento de la figura de la partera tiene que ver, según Towler y Bramall en su estudio sobre la historia de las comadronas, con el asentamiento de los antiguos pueblos nómadas durante el período neolítico, que trajo consigo cambios en su organización social, como el hecho de que las mujeres pertenecientes a la familia y luego a la comunidad sustituyeran a los hombres en la atención del parto. Las llamadas "mujeres experimentadas" se encargaron de esa labor excluyendo a los hombres durante los diez mil años siguientes (Towler y Bramall, 1997: 6).

En el transcurso de la historia podemos encontrar registros bíblicos sobre las comadronas en tres historias del Antiguo Testamento; en dos de ellas son consideradas como "diestras y valiosas profesionales" (Towler y Bramall, 1997:7). En la era egipcia la ocupación de matrona era reconocida, quien solía atender los 
nacimientos normales, incluidos los de la realeza (Towler y Bramall, 1997: 10). En el período griego existen pocas contribuciones a la obstetricia; sin embargo, en la época de Hipócrates y Sócrates (año 500 a.C.) se las reconocía socialmente y recibían honores, incluso estaban especializadas en dos grados, las de mayor experiencia y habilidad, quienes eran consultadas para los partos complicados, y las que atendían únicamente partos normales (Towler y Bramall, 1997: 14-15). La era romana acuna los conocimientos y prácticas heredadas de los egipcios a los griegos y de los griegos a los romanos, pero se sabe poco de las comadronas romanas, aunque se supone que en la era cristiana fue una profesión establecida y que requería personas educadas para su práctica (Towler y Bramall, 1997: 17).

Usandizaga (1944) no asegura que la profesión de las parteras tuviera reconocimiento oficial pero, por los testimonios sobre ellas en las referencias de las Cortes de Valladolid de 1523, infiere que debían tener cierta importancia social, aunque no las reconocieran legalmente. Por otra parte, en 1548, se promulga una ley para examinar a médicos, cirujanos, barberos y boticarios, y se prohíbe dicho examen a ensalmadores, parteras, especieros y drogueros. Las tentativas de mejorar el ejercicio profesional médico, dice el autor, se referían exclusivamente a médicos y cirujanos.

\section{Desplazamiento de las mujeres como sanadoras}

A finales del siglo XV la medicina se volvió una disciplina académica y una profesión establecida, con lo cual la exclusión de las mujeres se agudizó. Este movimiento coincidió con la ideología misógina circulante; en 1486, se publicó el tratado The malleus maleficarum, de Heinrich Kraemer y James Sprenger, el cual versaba sobre cómo identificar a las brujas y qué hacer con ellas. La persecución se capitalizó con la creencia generalizada en la inferioridad mental y espiritual de las mujeres, creencia que fue alimentada por la Iglesia (Reich, 1995: 2579). La exigencia de formación universitaria fue el vehículo para la exclusión legal de las mujeres de su práctica (Ehrenreich y English, 1988: 19). La descalificación de las mujeres sanadoras por parte de los profesionales médicos tuvo como primer blanco a sus pares, las médicas, posteriormente, el "arte de partear", en manos de las parteras por un par de siglos más (XVIII), sería otro campo fagocitado por la medicina oficial.

Por otra parte, Estados Unidos tiene una historia diferente puesto que, al haber relativamente pocos médicos universitarios en las colonias, las mujeres desempeñaron un papel importante como sanadoras, asumiendo el rol de médicas durante el siglo XVII. Sin embargo, algunas de ellas fueron denunciadas como brujas. En 1765 se puso en marcha la primera escuela de medicina en la Universidad de Pennsylvania, pero no aceptaba estudiantes mujeres. En el siglo XIX algunas mujeres, en casos excepcionales, fueron admitidas como estudiantes de medicina, pero se encontraban posteriormente con dificultades para ejercer. Por ejemplo, no eran admitidas para practicar o trabajar en los hospitales o eran rechazadas por las asociaciones médicas, lo cual les impedía el reconocimiento legal para ejercer. No es sino a partir de la segunda mitad del siglo XIX cuando se aprobó oficialmente que las mujeres tuvieran acceso a educación en medicina (Reich, 1995: 2579-2581).

\section{Desplazamiento de la partería o el arte de partear al ámbito de la biomedicina}

Antes del siglo XVI no era posible que un hombre fuera partero. Como la medicina y la cirugía aún se diferenciaban en los siglos XV y XVI, algunos barberos cirujanos empezaron a practicar el arte de partear. Si bien en un principio el partero o comadrón era "el cirujano que asiste a partos" para extraer un feto fallecido, detener un flujo copioso de sangre o practicar una cesárea después de morir la madre (Usandizaga, 1944: 217), en el siglo XVII se autorizó en París a los cirujanos para que asistieran partos normales.

Martínez y Pardo exponen que, en el siglo XVIII, la hasta entonces hegemonía de las parteras se puso sistemáticamente en cuestión "desde el poder político, intelectual y religioso, y [fue] sustituida de forma progresiva por la de los cirujanos comadrones" (2001:5). 
Los autores exponen que intervinieron diversos factores, entre ellos "los juicios que médicos, cirujanos y clérigos formularon sobre la incapacidad de las matronas", al denunciar casos de fallecimiento de madres y niños por iatrogenia, esgrimidos quizá con fines poblacionistas o humanitaristas. A propósito, Tomás Piferrer en su Compendio del arte de partear: compuestopara el uso de los Reales Colegios de Cirugía en 1765, apuntó lo siguiente:

\begin{abstract}
Entregada hasta el presente el Arte de partear a la poca instrucción de nuestras Comadres, es constante, que no ha recibido aumento, ni perfección. La ignorancia, inaplicación, y pocos medios, que han tenido para adelantarse, fueron la causa de tan lamentable atraso. Son generalmente las Parteras de una esfera, que haciéndoles emprender la dificultosa práctica de su facultad con poquísimas luces, que las dispongan, hace también que sean muy cortas las que les dan sus Maestras: Son vulgo, y como tales no recapacitan, porque les faltan principios, y si notan algo de extraordinario, más presto admiran el prodigio que buscan la razón, o el motivo de su rareza (Piferrer, 1765: II-III).
\end{abstract}

El discurso médico argumentó a favor de los cirujanos parteros el que, además de su habilidad manual, contaban con instrumental que sólo ellos poseían y sabían usar (Martínez-Pardo, 2001: 8). El siguiente paso fue la sanción legal. En 1750 el protomedicato instauró el examen para todas las parteras (Martínez-Pardo, 2001: 10; Usandizaga, 1944: 216) arguyendo "muchos malos sucesos en los partos, provenidos de la impericia de las mugeres llamadas parteras" (Martínez-Pardo, 2001). En la misma ley se reguló la asistencia de los cirujanos a los partos y los designó para ejercer el oficio de parteros por ser esta labor parte de la cirugía.

Otra cuestión que no puede dejarse de lado es que la partería, además, era un campo lucrativo (Martínez y Pardo 2001: 22). Ehrenreich y English (1988: 30) señalan que, en Estados Unidos, "en 1910 cerca del 50\% de los niños nacían con ayuda de una comadrona", con lo que se "desperdiciaban [...] casos de estudio" para la obstetricia, además de que "las mujeres pobres gastaban cerca de 5 millones de dólares anuales en comadronas, 5 millones que hubiesen ido a parar en cambio a los bolsillos de los 'profesionales"' (Ehrenreich y English, 1988).

Martínez y Pardo (2001: 13) destacan que el proceso "de subordinación y pérdida de hegemonía de las matronas" fue lento y complejo. Entre las razones para ello mencionan "los mecanismos de solidaridad femenina inherentes a la relación de las mujeres con la matrona, [...] la escasez de cirujanos parteros, la incapacidad de abarcar todo el territorio, la imposibilidad de la mayoría de pagarse un cirujano [o] la inaccesibilidad del mundo rural" (Ehrenreich y English, 1988: 12-13). En nuestra opinión, estas razones no son parte del pasado, sino que forman parte del presente de muchos países, principalmente en las regiones más empobrecidas del planeta, por lo que nos encontramos en un conflicto en el que no se sabe qué hacer con las parteras tradicionales y el que ellas se encuentren o no al margen del sistema sanitario se dirime a través del reconocimiento biomédico.

El parto atendido por parteras tradicionales no es una realidad anecdótica o residual en nuestros días. La cuestión es qué papel asignan los organismos internacionales a estos recursos de salud extraoficiales.

\section{Breve repaso histórico en México}

Al igual que en el caso europeo, desde la época colonial en México los médicos de formación universitaria han pretendido ser los únicos capacitados para proporcionar atención médica y han ejercido presión sobre el Estado para que legisle en este sentido (Carrillo, 1998: 158). En relación con las parteras tradicionales, desde esa época se les ha culpabilizado por la mortalidad maternoinfantil, al tiempo que se proclamaba la necesidad de su entrenamiento y licencia o su total reemplazo por doctores y enfermeras (Birn, 2006: 166). Así, al focalizarse en las parteras, se redujo la comprensión de las causas y soluciones de la alta mortalidad materno-infantil y se desarticuló este problema de los determinantes sociales y económicos y medicalizando a las parteras, el nacimiento y la maternidad misma (Birn, 2006: 137). 
A partir del siglo XIX la estrategia consistió en reemplazar a las parteras tradicionales por "mujeres alfabetas entrenadas en las escuelas de medicina, que fuesen auxiliares de los facultativos" (Carrillo, 1998: 158). A principios del siglo XX —fines de la década de los veinte - una nueva política pública sanitaria fue dirigida a las mujeres como curadoras, pacientes o trabajadoras de la salud, a través de las primeras unidades médicas, que atraían a las parteras para su "profesionalización" para que luego regresaran con un rol más profesionalizado, pero también más circunscrito a la arena médica clínica (Birn, 2006: 151-152). Las sesiones de entrenamiento estaban diseñadas para limitar el rol de las parteras a lo médico y para deshacerlas de sus funciones sociales y rituales. De esta manera, las parteras profesionales surgieron no sólo como una figura de apoyo a la profesión médica y subordinada a ella, sino también como "práctica represora de la actividad de las parteras tradicionales" que resultaba "competitiva de la medicina académica" (Carrillo, 1998: 159).

De acuerdo con Birn, las unidades médicas fueron ganando el territorio que previamente había sido del dominio de las mujeres al incluir el embarazo, el cuidado infantil y la partería entre sus competencias. Las unidades justificaron la invasión al relacionar la alta mortalidad infantil con la ignorancia materna y las prácticas erróneas de las parteras tradicionales, quienes atendían más del 90\% de los nacimientos (Birn, 2006: 162). En 1934 el reporte anual del Servicio de Higiene Rural se quejaba de que muchas mujeres, por la ignorancia o su economía, preferían a las parteras empíricas, quienes cobraban poco, lo que constituía un gran peligro tanto para las madres como para los niños. Por ello, se consideraba necesario identificarlas, monitorearlas y entrenarlas para "darles un sentido de responsabilidad y poner límites a su trabajo, para que no amenacen las vidas de las madres y sus hijos" (Birn, 2006: 162-163). En lugar de desplazar a las parteras, buscaron transformarlas en modernas puericultoras pagándoles para que acudieran semanalmente a "clubs de parteras”. En Cuernavaca, Morelos, por ejemplo, las parteras fueron presionadas para ir a clases para que "el doctor pudiera instruirlas en prácticas de nacimiento menos peligrosas". Este entrenamiento en obstetricia y partería, como un médico señaló, consistía principalmente en la instrucción de lo "que no debían hacer". ${ }^{4}$

El control no sólo sobre las parteras tradicionales, sino también sobre las profesionales, fue en aumento con la instauración de la ginecología como especialidad (Carrillo, 1998: 159).

\section{Medicalización del parto}

El ámbito de la asistencia a la reproducción - embarazo, parto y puerperio - por parte de la llamada medicina occidental obiomedicina es relativamente reciente - siglo XVIII- (Reich, 1995: 2579) debido a que el parto no era considerado un evento patológico y se relegaba a un plano "natural" y de mujeres.

Martínez y Pardo (2001: 5) señalan que en el Diccionario de autoridades, el primero de los publicados por la Real Academia Española de la Lengua en 1726, fueron definidas las palabras "comadre" y "partera", y no así "partero" y "comadrón", como el oficio encargado de "asistir y ayudar a parir". Por otro lado, apuntan que la voz "partero" se popularizó en castellano en las primeras décadas del siglo XVIII. En el mismo diccionario se diferencia a éste de la partera al definirlo como "el cirujano que asiste a los partos". Hoy en día se sigue manteniendo esta distinción. La Academia da dos acepciones a la palabra partera: la primera que admite masculino se refiere al ejercicio profesional de la partería, mientras que la segunda se refiere a la partera tradicional y sólo se utiliza en femenino. ${ }^{5}$

En la actualidad, el concepto de partera es polisémico y admite variaciones como parteras empíricas, parteras tradicionales, comadronas, comadres o matronas, y los significados de cada una de estas expresiones pueden diferir dependiendo de quién los utilice. Así, los organismos internacionales de salud a lo largo del tiempo han transformado la manera de nombrarlas, sin explicitar las razones del cambio conceptual (OMS, 1966; UNFPA, 2002). El Informe anual 2008 del Fondo de Población de las Naciones Unidas señala lo siguiente: 
El UNFPA y la Confederación Internacional de Comadronas emprendieron un programa a fin de acrecentar el número de partos atendidos por parteras en los países en desarrollo. La iniciativa se centrará en establecer una fuerza laboral sostenible de partería y reforzar la educación y los estándares de la profesión de partera como parte de acciones más amplias encaminadas a fortalecer los sistemas de salud en 11 países (UNFPA, 2009: 2).

No se aclara si, al referirse a que un mayor número de partos sean atendidos por parteras, se está refiriendo a parteras capacitadas o calificadas: "El UNFPA ya está ayudando a los países a mejorar sus clínicas y hospitales al servicio de las embarazadas, a capacitar a las parteras y otro personal médico" (UNFPA, 2009: 2).

En los últimos años las parteras tradicionales ${ }^{6}$ han ido desapareciendo de los informes de los organismos internacionales. Es más, cuando se habla de parteras generalmente se hace referencia a las parteras profesionales y se invisibiliza la existencia de parteras tradicionales. En Elestado de las parterasen el mundo de 2011 se define a las parteras tradicionales como

[...] un grupo heterogéneo de miembros de la comunidad que no han recibido formación académica, que se desempeñan independientemente de los servicios de salud pública y que proporcionan atención durante el embarazo, el parto y el período postnatal (UNFPA, 20llb: 10).

\section{¿Un mundo de parto medicalizado?}

Hablar de parteras o comadronas tradicionales puede parecer, para algunos, algo anecdótico en un mundo cada vez más medicalizado y en el que la tecnología en el parto pretende ser la norma. Lo cierto es que, en 2007, el 37\% de los partos no fue atendido por personal calificado. ${ }^{7}$ Aunque en ese año la cobertura superó el 99\% en países desarrollados, en los países de bajos ingresos fue inferior al 60\% (OMS, 2007a) y en 2009 sólo ascendió al 66\% (UNFPA, 2011a: 115). ${ }^{8}$ En 2012, en los países desarrollados casi todos los partos eran atendidos por personal calificado, pero en los países de bajos ingresos la proporción fue del 65\%. No obstante, el panorama es heterogéneo y en algunos de los países más empobrecidos esa proporción desciende a menos del 20\% (UNFPA, 2012). El resto recibió atención de las parteras tradicionales, familiares o vecinos, o no recibió ninguna atención. Evidentemente, este dato no nos dice mucho sobre las enormes diferencias entre las distintas regiones, países o al interior de un mismo país entre sus zonas rurales y urbanas, grupos étnicos, etcétera.

Mientras en las "regiones más desarrolladas" (UNFPA, 1996: 76) el 99\% de los partos recibió atención por personal calificado (UNFPA, 2011b: 115), este porcentaje baja al 63\% (UNFPA, 201lb: 115) si nos referimos a las "regiones menos desarrolladas"10 (UNFPA, 1996:76) y al 39\% (UNFPA, 201lb: 115) en el caso de los "países menos adelantados"."

Aunque ésta es la panorámica que muestran los datos oficiales, es difícil saber realmente cuántos partos son atendidos por personal calificado. En primer lugar, no todos los países disponen de sistemas de registro civil y, en la mayoría de los que cuentan con él, tan sólo se registra la cuarta parte de los nacidos vivos (UNICEF, 2009). Los partos atendidos por personal calificado en los hospitales, centros de salud o en domicilios son registrados y forman parte de los informes mensuales de atención de los que se nutren las estadísticas nacionales. No ocurre así con los partos atendidos fuera del sistema de salud. Sólo una minoría de las parteras tradicionales reporta a los centros de salud los partos que atiende. Por ello, habitualmente se trabaja con estimaciones basadas en los partos esperados para una determinada región; los partos no atendidos por personal calificado son el resultado de restar los partos atendidos por personal calificado a los partos esperados.

Por otra parte, el término "personal calificado" tal vez no refleje con claridad en qué medida las mujeres disponen efectivamente de acceso a la debida atención, particularmente cuando surgen complicaciones. Uno de los principales problemas es determinar qué se entiende por personal calificado. Aun cuando se ha tratado de dar una definición uniforme de médico, enfermera, partera y partera auxiliar, es probable que 
muchos profesionales de la salud que se catalogan como "calificados" no cumplan los criterios aceptados internacionalmente (UNFPA, 2004b: 13). En ocasiones, es el personal auxiliar el que atiende los partos y el personal calificado - partera profesional, enfermera diplomada o médico- sólo atiende los "partos complicados" en el mejor de los casos. Este personal auxiliar no se incluye en la definición de personal calificado y el UNFPA, en sus últimos documentos, los sitúa en el mismo plano de competencia que a las parteras tradicionales.

No es extraño que existan dificultades para la clasificación del personal como calificado o no. El concepto ha sufrido modificaciones en los últimos tiempos que coinciden con el cambio de paradigma que, desde la década de los noventa, va abandonando la capacitación de las parteras tradicionales como estrategia para reducir la mortalidad materna. Para empezar, el propio término "personal calificado" es reciente y, por períodos, ha sustituido al de "personal capacitado" que anteriormente se utilizaba. Asimismo, se han modificado los criterios de inclusión o exclusión y se ha hecho más hincapié en la formación médica del personal. En el documento de UNFPA sobre mortalidad materna del 2002 se utiliza el concepto de "personal capacitado":

El concepto de atención por personal capacitado denota la existencia de agentes de salud que han recibido formación profesional y adquirido los conocimientos prácticos necesarios para atender un parto normal y diagnosticar las complicaciones obstétricas o remitir esas pacientes a establecimientos de mayor capacidad. Esos agentes de salud son por lo general médicos, parteras o enfermeras. [...] No se incluyen en esta categoría las comadronas y parteras tradicionales, hayan o no recibido capacitación (UNFPA, 2002: 9).

Dos años más tarde, el término utilizado es ya el de "personal calificado", que se define como

[...] un trabajador de la salud con formación médica y conocimientos sobre atención del parto (partera, enfermera diplomada o médico), que posee las aptitudes profesionales necesarias para atender partos normales y encargarse del diagnóstico, el tratamiento o la remisión a otros establecimientos de los casos de complicación obstétrica (UNFPA, 2004a: 7).

En años posteriores se utiliza en algunos documentos el adjetivo "capacitado", en otros "calificado", o incluso ambos indistintamente en el mismo documento. En el informe de UNFPA, Estado de la población mundial 2008, aparece una nueva definición:

El personal de partería calificado está constituido por profesionales de la salud acreditados - parteras, médicos o enfermeras- y dotados de conocimientos para atender embarazos y partos normales (sin complicaciones) y el período postnatal inmediato; detectar y tratar complicaciones en la mujer y el recién nacido; y remitir a las pacientes a servicios apropiados de atención obstétrica y de emergencia. Esta definición excluye a las parteras tradicionales, dotadas o no de capacitación (UNFPA, 2008).

El propio Fondo de Población reconoce la confusión creada con estos términos:

Durante los primeros diez años del presente siglo, en un esfuerzo por revitalizar la atención primaria de la salud, muchos países han impartido capacitación a nuevos tipos de agentes de salud con diversas denominaciones: enfermeras auxiliares, parteras comunitarias, agentes de difusión de salud y personal comunitario de partería. Esos profesionales han recibido formación en diversos conjuntos de aptitudes, con diferentes currículos y en cursos de capacitación de diferente longitud. Por otra parte, tal vez muchos de esos profesionales se individualicen y denominen, por inadvertencia, personal de partería cualificado, sin haber satisfecho los requisitos indicados en la definición de la OMS. De esos profesionales de la salud, son pocos los que han recibido capacitación para adquirir plenamente todas las competencias de una partera y no se han establecido entre diferentes 
países acuerdos sobre procesos estandarizados de educación ni de reglamentación u otorgamiento de licencias. El resultado es una creciente confusión acerca de quién es un profesional de partería cualificado (UNFPA, 201lb: 4).

Otro factor que influye en que la atención del parto por personal calificado esté sobredimensionada es la presión que se ejerce por el cumplimiento de metas sobre el personal de salud en los países en desarrollo. El parto atendido por personal calificado figura como indicador para medir la reducción de la mortalidad materna en la Conferencia Internacional sobre la Población y el Desarrollo de El Cairo (Naciones Unidas, 1994: 54), ${ }^{12}$ en los Objetivos de Desarrollo del Milenio ${ }^{13}$ (Naciones Unidas, 2000) o en la iniciativa HIPC del Banco Mundial y del Fondo Monetario Internacional para medir el progreso de la condonación de la deuda para los Países Pobres Altamente Endeudados (OXFAM, 2005; Colom, 2003). ${ }^{14}$ Estas presiones pueden haber influido sobre el personal de salud, cuyo trabajo es evaluado por el cumplimiento de estos indicadores, un "incentivo perverso" para la sobrestimación de partos atendidos por personal calificado.

\section{Transformación del discurso respecto a las parteras, desde los organismos internacionales de salud}

Como ya se comentó, se observa un cambio de paradigma a partir de los noventa, cuando no se consideró la capacitación de las parteras tradicionales como una estrategia adecuada para disminuir la mortalidad materna. Esto se fue develando en las definiciones de partera tradicional que surgieron, donde se resaltó lo negativo, lo que no fue o lo que desconocía:

Las parteras tradicionales carecen de capacitación médica académica, aun cuando algunas han recibido capacitación para evitar prácticas peligrosas o contaminantes (UNFPA, 2002b: 22).

Las parteras tradicionales no tienen instrucción suficiente ni los conocimientos necesarios para atender complicaciones obstétricas o remitir esos casos a establecimientos adecuados (UNFPA, 2004b: 7).

La carencia de conocimientos es tan intrínseca a su condición que, por definición, se las considera incapaces de adquirirlos:

Las parteras tradicionales, hayan o no recibido capacitación, carecen de los conocimientos necesarios para atender problemas que amenazan la vida de la embarazada (UNFPA, 2002: 12).

En ocasiones no se distingue entre partera tradicional y otras personas que puedan atender ocasionalmente un parto; de esta forma, se niegan sus conocimientos y experiencia. ${ }^{15}$

En algunos casos, la partera tradicional tiene una tarea claramente definida; en otros casos, es sólo una parienta que ayuda a los miembros de la familia (UNFPA, 2002: 12).

Una partera tradicional [Traditional Birth Attendant, TBA] es una persona que vive en la comunidad y atiende el embarazo y el parto (UNFPA, 2004b: 7). [...] el UNFPA proporciona botiquines para el parto aséptico que pueden ser utilizados por parteras, miembros de la familia o incluso parteras tradicionales en situaciones de emergencia, a fin de minimizar el riesgo de infección (UNFPA, 2002: 13).

En los últimos años existe un acuerdo en que la prioridad es que personal calificado atienda a todas las embarazadas, pero en este concepto no se incluye a las parteras tradicionales. El motivo principal para ello es el abandono del "enfoque de riesgo" —que promovía la atención prenatal para detectar los embarazos de alto riesgo y asegurar su atención especializadapara dar paso a un "enfoque basado en los derechos humanos", en el que se considera que todas las embarazadas corren riesgos y tienen derecho al acceso a una atención de su parto por parte de personal calificado. El problema es que, por múltiples causas, un parto atendido por personal calificado aún no es 
una posibilidad real para la mayoría de las mujeres en los países en desarrollo. Muchas son las causas de esta situación: una es simplemente la escasez de agentes de salud profesionales capacitados y otra es, cuando los hay, su deficiente distribución geográfica: hay una escasez crónica de profesionales en las comunidades pobres y rurales de los países en desarrollo (UNFPA, 1988: 12), pues, en su mayoría, dichos profesionales prefieren permanecer en zonas urbanas (UNFPA, 2002: 14). Además, se ignora la importancia de la disponibilidad de infraestructura y redes para resolver las complicaciones.

Durante años la opción fue capacitar a las parteras como estrategia para reducir la mortalidad materna. En los años setenta se generalizó la formación de las parteras tradicionales con el propósito de mejorar los servicios obstétricos en los ámbitos en los que faltara personal sanitario profesional y allí donde los hospitales no dispusieran de camas o de personal suficiente para que todas las mujeres pudieran dar a luz en ellos. Estas parteras ya existían y atendían a las parturientas generalmente en zonas rurales-, eran accesibles y aceptables desde el punto de vista cultural, e influían en las decisiones de las mujeres respecto al uso de los servicios de salud. Se consideró que formarlas en los métodos modernos de alumbramiento era una nueva forma de progresar (OMS, 2005a: 74). Esta actitud partía de una definición de la partera tradicional diferente, en la que se reconocía sus conocimientos y experiencia y su papel como elemento fundamental dentro de la comunidad.

En numerosos países, muchas mujeres dan a luz sólo con la asistencia de comadronas tradicionales. Son estas personas a veces iletradas y que con frecuencia carecen de toda instrucción obstétrica pero que conocen bien las costumbres populares en cuanto se refiere a los cuidados que han de darse a la madre y al niño, y suelen ser uno de los miembros más respetados de la colectividad (OMS, 1996: 17).

El discurso se mantuvo sin demasiadas variaciones hasta finales de los años noventa. Durante este tiempo se realizaron diversas investigaciones y estudios para evaluar la eficacia de estas intervenciones. ${ }^{16}$ En el informe de la 25ํㅡㄹ Conferencia Sanitaria Panamericana de 1998 de la Organización Panamericana de la Salud se seguía defendiendo la eficacia de esta estrategia:

En Guatemala, un programa patrocinado por el Ministerio de Salud y el Instituto de Nutrición de Centro América y Panamá (INCAP) se propuso específicamente reducir la mortalidad materna y neonatal. [...] Las metas eran mejorar los conocimientos y el desempeño de las parteras tradicionales, recurso al alcance de las 500.000 personas de la población interesada, y humanizar el trato de estas parteras y de sus pacientes en el hospital y los centros de salud, a fin de establecer un vínculo entre los elementos formal y no formal del sistema. [...] Los resultados son notables. [...] Hoy día, 10 años después de haberse instaurado, el modelo sigue dando resultados pues otros donantes lo están adoptando, y su alcance se ha ampliado de modo que cubre a más de dos millones de personas a las que a menudo es difícil atender (OPS, 1988: 17).

En la siguiente conferencia de la OPS, en el año 2002, a pesar del cambio de paradigma y de considerar la atención del parto por personal calificado como la estrategia fundamental, no se descarta continuar capacitando a las parteras tradicionales. Las críticas se dirigen no a la pertinencia de la estrategia, sino a las fallas en su puesta en práctica, que no tienen que ver con la capacidad de la propia partera, sino con su articulación con el sistema. Debe recalcarse que el adiestramiento de parteras tradicionales, por sí solo, a falta de un sistema eficaz de referencia de pacientes y del apoyo adecuado de personal capacitado, no es suficiente para reducir la mortalidad materna. Cuando se emprende el adiestramiento de parteras tradicionales, éste tiene que formar parte de una infraestructura integral que incluya un sistema de referencia de pacientes, de supervisión y de evaluación (OPS, 2002: 9). Por su parte, UNFPA mencionaba algo similar en su informe de 1998: 
Ahora se reconoce que las parteras tradicionales no pueden, por sí solas, reducir sustancialmente la mortalidad derivada de la maternidad. Si se quiere que participen en la atención médica de la madre, es preciso crear o reforzar los vínculos entre ellas y el sistema de servicios públicos de salud (UNFPA, 1988: 28-29).

En virtud de esto se redefinen las funciones que la partera tradicional puede desempeñar sin riesgo, como el seguimiento de nacimientos, muertes y embarazos en la comunidad; la promoción de los servicios de salud, el apoyo a la familia mientras la mujer está ausente para dar a luz y servir de vínculo entre los servicios de salud y la comunidad (UNFPA, 2004b: 22).

En cambio, en la crítica a la capacitación de parteras tradicionales que hizo la OMS en el Informe sobre la salud en el mundo 2005, se cuestionó la propia estrategia en sí:

Los datos demostraron que la formación de las parteras tradicionales había repercutido poco en la mortalidad materna. [...] En la mayoría de los ámbitos es poco realista suponer que un curso de formación pueda tener algún efecto en la mortalidad materna. [...] Se está generalizando la impresión de que la estrategia ha sido un fracaso. Habremos tardado más de 20 años en darnos cuenta de ello y, a la postre, quién sabe si el dinero gastado no se habría empleado mejor en formar a parteras profesionales (OMS, 2005: 74, UNFPA 2011b: 10).

El discurso se ha vuelto paulatinamente contrario a la capacitación de parteras tradicionales y los últimos documentos abogan por el abandono de la estrategia. Se cuestionan también los contenidos de los programas de capacitación. Hay que destacar que la expresión "programa de capacitación" incluye una gran variedad de temáticas, metodologías, duración, periodicidad, evaluación y seguimiento. En nuestra opinión, este cuestionamiento no supone una objeción a la estrategia en sí, sino a la forma en que se ha materializado al aplicarse fórmulas generales y no adaptadas a la realidad de cada contexto.
La función, los conocimientos y la experiencia de las parteras tradicionales varían mucho de unas regiones a otras, e incluso dentro de un mismo país. No es, pues, técnicamente válido elaborar una estrategia general de formación sin tener en cuenta estas diferencias. [...] Un segundo problema es cualitativo: no está claro qué se les debería enseñar a las parteras tradicionales. Para modificar su forma de actuar es necesario comprenderla, algo que raras veces se ha sugerido. Aun cuando fuera posible cambiar algunos de los componentes de los conocimientos tradicionales, ello podría «desestabilizar» el conjunto (OMS, 2005: 74).

Por otro lado, se objeta la conveniencia de aumentar la supervisión y el apoyo a las parteras por la escasez de tiempo y recursos del personal sanitario (OMS, 2005). A nuestro entender, este reconocimiento de la falta de profesionales sanitarios contrasta con la apuesta de promover la atención por personal calificado que supone, obviamente, aumentar los recursos humanos en las zonas más alejadas.

Otro de los argumentos es la gran distancia del paradigma universalista biomédico propio del personal de salud frente a las culturas locales de las parteras tradicionales:

El rol social de las parteras tradicionales, como el de los curanderos, está profundamente enraizado en la cultura local. No se reduce a dispensar asistencia para una afección determinada: lo abarca todo y reinterpreta el padecimiento de la paciente en su contexto cultural. Los defensores de la estrategia de formación de las parteras tradicionales no han tenido presente la enorme brecha cultural que separa a los métodos asistenciales modernos de las actividades de las parteras tradicionales (OMS, 2005).

Es curioso que el enraizamiento de las parteras en la cultura local sea utilizado como argumento en contra de la eficacia de la capacitación de las parteras tradicionales, cuando ha sido uno de los argumentos a favor de la necesidad de trabajar con ellas. 
Algunos programas realizados más recientemente han tratado de promover el papel de las parteras tradicionales, dotadas de sensibilidad cultural, para que establezcan vínculos entre el sistema de salud y la comunidad. Los procedimientos médicos y la administración de medicamentos deben estar a cargo de profesionales de la salud capacitados. Por otra parte, muchas parteras y muchos médicos no han recibido formación en cuanto a los sistemas de creencias, la comunicación y la organización de las comunidades y en este aspecto, las parteras (o comadronas) tradicionales pueden ser más eficaces (UNFPA, 2002: 12).

Muchas mujeres comunican que los profesionales del sistema de salud oficial no son amables, ni comprenden sus valores y creencias culturales. En consecuencia, muchas mujeres acuden a miembros de su familia o a parteras tradicionales para la atención prenatal, del parto y del puerperio (OPS, 2002: 18).

En cambio, en el mencionado Informe sobre la salud del 2005, la OMS sostiene que son los "profesionales capacitados" los más apropiados para brindar una atención del parto culturalmente adecuada:

Los rituales que rodean al parto tienen su valor, así como el hecho de mantenerlos como un elemento básico de la vida familiar. Es lógico, pues, que se necesite y se demande una atención cercana a los hogares y las formas de vida de las personas, y cercana también a sus costumbres en lo relativo al alumbramiento [...] Estas intervenciones sólo pueden dispensarlas profesionales en posesión de una serie de conocimientos prácticos y competencias integrados y a quienes se denomina de forma abreviada «asistentes de partería calificados» (OMS, 2005: 75-76).

A pesar de todo, no se ofrece una explicación de qué es lo que ha cambiado para considerar que los médicos y las parteras profesionales estén ahora dotados de mayor sensibilidad cultural, de forma que ya no sea necesario fomentar el papel de la partera tradicional como vínculo entre el sistema de salud y la comunidad.
Uno de los principales argumentos a favor de la estrategia de capacitación de parteras tradicionales era su liderazgo dentro de la comunidad. La capacitación en el reconocimiento de los signos de peligro en el embarazo, parto y puerperio, junto con su integración al sistema de salud o el fortalecimiento de sus vínculos con él, suponía una oportunidad para convencer a las mujeres de que acudieran a los hospitales cuando se presentaran complicaciones:

En lugares donde las mujeres normalmente dan a luz en casa con ayuda de una partera, los investigadores recomiendan ahora capacitar a esta última para que sepa reconocer las complicaciones mortales y remitir a las mujeres a recibir atención urgente de proveedores capacitados (Ransom y Yinger, 2002: 11).

El Fondo apoya a las parteras tradicionales para que tengan un papel de enlace entre las comunidades y el personal de salud capacitado. Las parteras tradicionales pueden motivar a las mujeres a que utilicen servicios prenatales y de planificación de la familia y cuando surgen complicaciones, pueden destacar la necesidad de que las mujeres obtengan Atención Obstétrica de Emergencia en los hospitales y otros establecimientos de salud (UNFPA, 2002: 13). La opinión generalizada es que las parteras tradicionales pueden ser paladines de la atención por personal calificado y alentar a las mujeres a que acudan a los servicios de salud que cuentan con la debida capacitación médica. [...] Ahora bien, es preciso apoyar la función de las parteras tradicionales como un nexo entre las mujeres y el sistema de atención de la salud. Cuando se necesite una remisión a un establecimiento mejor preparado, las parteras tradicionales deberían acompañar a las mujeres hasta dicho establecimiento y ser bien recibidas allí por los profesionales de la salud (UNFPA, 2004b: 2l).

Sin embargo, a juzgar por el informe de 2005 de la OMS, la estrategia tampoco ha sido eficaz en este sentido, aunque cabe preguntarse si el abandono de ésta mejorará la actitud de las parteras tradicionales con respecto al sistema oficial de salud. 
Por último, mientras que algunos especialistas confían en que las parteras tradicionales ayuden al menos a persuadir a las mujeres con complicaciones para que acudan a los hospitales, otros señalan exactamente lo contrario; es decir, que las parteras tienden a demorar la partida al hospital e incluso a disuadir deliberadamente a las mujeres de acudir a él (OMS, 2005: 74). En 2011, de nuevo pareció apreciarse un cambio en el discurso. El informe Estado de la partería en el mundo 2011 abrió la posibilidad de que se pudiera trabajar con las parteras tradicionales como aliadas para el sistema de salud, pero con la condición de que reconocieran y aceptaran su posición de subordinación al sistema biomédico.

Hay pruebas objetivas de que, en determinadas circunstancias, la capacitación de las parteras tradicionales puede mejorar conocimientos y actitudes, y conducir a reducciones de pequeña magnitud, aunque apreciables, en la mortalidad perinatal. Pero no hay pruebas concluyentes de que esta capacitación sea eficaz en función de los costos, ni de que tenga efectos directos de reducción de la cantidad de defunciones de mujeres en el embarazo o el parto (UNFPA, 201lb: 10).

Las parteras tradicionales deberían sólo alentar la atención del parto por personal calificado:

Dado que las parteras tradicionales tienen cercanía cultural con las mujeres, están en condiciones incomparables para motivar y ayudar a las mujeres y a sus familias a planificar la atención del parto por un agente de salud cualificado. A condición de que la partera tradicional tenga en claro sus funciones y responsabilidades, y de que haya entablado una relación positiva con los demás miembros del personal de partería, es posible esperar resultados beneficiosos (UNFPA, 201lb: 10).

Con todo, parece que los organismos internacionales no acaban de definir cuál es la estrategia o qué papel les corresponde a las parteras tradicionales. La pregunta no es tanto por qué se hace una apuesta por la atención por personal calificado como la opción más eficaz, sino por qué se decide que no es necesario realizar un trabajo conjunto con las parteras tradicionales como posibles y deseables aliadas estratégicas del sistema de salud, sobre todo allá donde este último se muestra incapaz de alcanzar a toda la población. Esta postura es la que lleva a minimizar la importancia de las parteras, ${ }^{17}$ de sus conocimientos, de sus acciones y de su influencia sobre las decisiones de salud en la población, de igual forma como se hace con otros agentes sanitarios externos al sistema oficial.

Es indiscutible la relación entre reducción de mortalidad materna y atención del parto por personal calificado, y que tanto la mortalidad materna como la neonatal son bajas en los países en los que las parturientas reciben atención profesional competente, con los equipos, medicamentos y demás suministros necesarios para tratar de forma eficaz y oportuna las complicaciones (OMS, 2005: 70). Pero también es cierto que otros factores influyen en la misma medida en esta mortalidad y que, en su mayoría, los países que han alcanzado mejores cifras en el parto atendido por personal calificado también cuentan con mejores condiciones higiénico-sanitarias en general y con un mejor estado nutricional y de salud de las madres. Hay multitud de factores que influyen en la mortalidad materna. Esto se expresa en las diferencias que se observan en las tasas de mortalidad materna entre países con similares cifras de parto atendido por personal institucional. Éste es el caso, por ejemplo, de Guatemala y Sierra Leona, con cifras de atención por personal cualificado en torno a un $40 \%-41 \%$ y $42 \%$ respectivamente - y razón de mortalidad materna (RMM) de 110 y 970, o de Bangladesh - 18\%- y Afganistán - 14\% - en atención cualificada del parto y una RMM de 340 y 1400. Es importante señalar que Guatemala y Bangladesh cuentan con algunas de las experiencias más exitosas en el trabajo de capacitación e integración de parteras tradicionales en los sistemas de salud. ${ }^{18}$

Un argumento a favor de la estrategia de formación de las parteras tradicionales, en sus inicios, era el 
económico: las parteras tradicionales reciben su remuneración de las mujeres y sus familias, y por esa razón no pesan sobre el presupuesto público (UNFPA, 2002b: 22). El auge de los programas de capacitación de parteras tradicionales se da en los años setenta, la década de Alma Ata y del lema "salud para todos en el año 2000". En un momento en que el objetivo de los Estados debía ser asegurar la prestación de atención de salud básica para toda la población, "liberar" al presupuesto público se veía con buenos ojos. Podríamos estar viviendo, desafortunadamente, el declive de un enfoque del derecho a la salud para dar paso a visiones más mercantilistas. Por eso, tal vez, en nuestro tiempo de políticas de salud de recuperación de costos y paulatina privatización de los servicios de salud, las antiguas aliadas pueden convertirse en competidoras por el mercado del parto. Esto explicaría las contradicciones entre el discurso y la práctica y por qué, a pesar de calificar como ineficaz la estrategia de capacitación de comadronas, se siguen impulsando programas con este enfoque para las áreas de menores recursos.

\section{Conclusiones}

A pesar de la evolución del discurso de los organismos internaciones que dictan las políticas sanitarias respecto a la salud reproductiva —UNFPA y OMS-, desde la década de los noventa una constante ha sido considerar que las parteras tradicionales no pueden llegar a ser "profesionales de la salud," aunque tengan las habilidades y conocimientos para ser consideradas como "capacitadas". Esto ocasiona la reconfiguración de las posiciones en cuanto a la delimitación de funciones tanto de la partera tradicional y su relación con el sistema de salud, como del llamado personal calificado.

El discurso se ha radicalizado hacia la colocación de las parteras como figuras externas al sistema de salud y cuya proximidad dependerá de su subordinación a los dictados de la biomedicina. Si bien en la actualidad estamos en un tiempo de corrección política en el lenguaje, el paralelismo entre los discursos de descalificación de la partera del siglo XVIII y de la actualidad es evidente. Sorprende la persistencia en considerarlas incapaces de aprender las habilidades necesarias a pesar de que se les "instruya," sobre todo porque es un recurso humano al que los ministerios de salud recurren en aquellos lugares con insuficiente personal calificado. Se puede observar en los cambios de discurso un proceso que aún está en marcha y que revela la diferencia de visiones al interior de los organismos de salud y entre ellos. Estas divergencias revelan lo caótico del proceso y lo confuso que resulta enfrentar sus conflictos, lo cual podemos constatar, por ejemplo, en la polisemia de términos para nombrar tanto a las parteras como al personal "profesional" de la partería.

En la Ilustración se hizo evidente el desplazamiento progresivo de la partera por el cirujano obstetra. Esta puerta de entrada se amplió con la atención de una vasta diversidad de complicaciones hasta conseguir abarcar los partos eutócicos. De forma similar, en el discurso de las agencias internacionales se comenzó por cuestionar que la partera fuera el personal adecuado para atender las complicaciones; se continuó poniendo en tela de juicio su capacidad para atender aquellos que no las tuvieran, y se terminó por excluirlas, incluso, de su papel de puente con el sistema sanitario, a pesar de ser personas socialmente significativas en la comunidad.

Asimismo, es innegable que, en este proceso histórico de desplazamiento de la partería, por encima de justificaciones sobre la "(in)capacidad" de las parteras, se libró una lucha por un espacio laboral, económico y de reconocimiento social entre una profesión antigua y una emergente. En el siglo XVIII hubo razones económicas y no sólo técnicas, y planteamos nuestra duda de si algo similar podría estar ocurriendo actualmente.

\section{Notas}

${ }^{1}$ Para las autoras el discurso se refiere a los conceptos o definiciones sobre las tareas, desempeño y limitaciones de la partería tradicional y del personal calificado expresados en los principales documentos -informes, evaluaciones - publicados por los organismos que 
dictan la política pública internacional sobre salud materna. Estos documentos pretenden estar basados solamente en evidencia científica biomédica pero, como todo escrito, reflejan la ideología dominante en dichos organismos.

2 De acuerdo con Rodríguez et al. (2012: 1), la medicalizacion "es el fenómeno dinámico dela extensión continua del dominio y la autoridad de la medicina a todos los órdenes de la vida social. La medicalización se da cuando un fenómeno social, un aspecto de la vida cotidiana que hasta el momento no había formado parte de la esfera médica empieza a ser traducido en estos términos".

3 Fondo de las Naciones Unidas para la Población, Organización Mundial de la Salud y Organización Panamericana de la Salud.

${ }^{4}$ Esta visión permaneció largo tiempo. Cuarenta años después, en 1973, el responsable del programa de entrenamiento de parteras de la Secretaría de Salud decía "sobre todo, ellas deben ser instruidas específicamente en lo que no deben hacer" (Birn, 2006: 166).

${ }^{5}$ partera/ro:l. m. y f. Persona con títulos legales que asiste a la parturienta. 2. f. Mujer que, sin tener estudios o titulación, ayuda o asiste a la parturienta.

6 En adelante utilizaremos el término "parteras tradicionales" para distinguirlas de las parteras profesionales, que son usualmente enfermeras especializadas en la atención del parto. Aunque no lo consideremos el término idóneo, es el que se emplea en los documentos oficiales y, cuando se refieren simplemente a partera, están hablando de personal con formación médica.

7 La Declaración del Milenio sobre Desarrollo también como conocida como Objetivos de Desarrollo del Milenio, firmada en septiembre de 2000 por los países miembros de Naciones Unidas-, en la que estableció como un indicador de progreso del quinto objetivo mejorar la salud materna y la proporción de partos atendidos por personal calificado, tiene como antecedente importante la declaración conjunta sobre reducción de la mortalidad materna de la OMS, el UNFPA, el Fondo de las Naciones Unidas para la Infancia (UNICEF) y el Banco Mundial en 1999. En ella se hizo referencia a la atención del parto por personal sanitario calificado - partera calificada o médicocomo un indicador sensible de la mejora en la atención del parto y recomendaba su utilización como elemento central de cualquier política o programa encaminado a la reducción de las muertes maternas (Naciones Unidas, 1999).

${ }^{8}$ Con base en datos del sitio web de los indicadores de los objetivos de desarrollo del milenio - hhtp://mdgs. un.org/unsd/mdg/. Naciones Unidas, Departamento de Asuntos Económicos y Sociales, División de Estadística-. Los datos son las estimaciones más recientes de que se supone, posteriores a 1995. "Los partos atendidos por personal cualificado indican el porcentaje de partos con atención de personal capacitado para: proporcionar atención obstétrica que salva vidas, inclusive la necesaria supervisión, atención e instrucción a las mujeres durante el embarazo, trabajo de parto y el periodo postparto; atender el parto sin ayuda y por sí mismos; cuidar al recién nacido. No se incluye a las parteras tradicionales, aun cuando hayan recibido un breve curso de capacitación" (UNFPA, 201lb).

9 Norte América, Japón, Europa, Australia y Nueva Zelanda.

${ }^{10}$ África, Latinoamérica y el Caribe, Asia -exceptuando Japón-, Melanesia, Micronesia y Polinesia.

${ }^{11}$ Afganistán, Angola, Bangladesh, Benin, Bhután, Burkina Faso, Burundi, Cabo Verde, Camboya, Chad, Comoras, Djibouti, Eritrea, Etiopía, Gambia, Guinea, Guinea Ecuatorial, Guinea-Bissau, Haití, Islas Salomón, Kiribati, Lesotho, Liberia, Madagascar, Malawi, Mauritania, Malí, Mozambique, Myanmar, Nepal, Níger, Republica Centroafricana, República Democrática del Congo, República Democrática Popular de Lao, República Unida de Tanzania, Rwanda, Samoa, Santo Tomé y Puerto Príncipe, Somalia, Sudán, Togo, Uganda, Vanuatu, Yemen y Zambia. www.cinu.org, Naciones Unidas. Centro de información México, Cuba y República Dominicana

${ }^{12}$ Párrafo 8.22: "Todos los nacimientos deberían contar con la asistencia de personas capacitadas, de preferencia enfermeras y parteras, pero al menos comadronas capacitadas". 
${ }^{13}$ Naciones Unidas, Objetivos de Desarrollo del Milenio, objetivo 5 , meta 6 , indicador 17.

${ }^{14}$ La iniciativa HIPC tuvo como finalidad reducir las cargas excesivas de la deuda que afectaba a los países más pobres del mundo. La iniciativa comprendió tres etapas distintas. En la tercera y última etapa, los países tuvieron que elaborar un DLEP (documento estratégico de lucha contra la pobreza) completo, con base en consultas con la sociedad civil. Esta iniciativa supuso una alternativa a los desprestigiados créditos del Servicio Reforzado de Ajuste Estructural (SRAE), que pasan a nombrarse Servicio de Crecimiento y Reducción de la Pobreza. Los SRAE se criticaban por su alta condicionalidad en materia de política económica. Esta obligación es la que se llama condicionalidad macroeconómica. La novedad más importante radica en el hecho de que se pretende que las políticas de lucha contra la pobreza pasen a ser el centro de la estrategia económica del país. En la mayoría de los DELP la atención de parto por personal cualificado figura como uno de los indicadores que verifican el cumplimiento de los objetivos.

${ }^{15}$ En este sentido es curioso que el término "partera empírica" haya desaparecido de los documentos de los organismos internacionales para pasar a ser denominadas parteras tradicionales.

16 Ver como ejemplos Mangay-Manglacas y Pizurki (1981), Araujo (1984) y O’Rourke (1995).

17 Subyace a dicha minimización o marginalidad la intersección de diferentes dimensiones culturales tales como la etnia, el género o la clase social, que interactúan entre sí en diferentes niveles y simultáneamente, por lo que, parafraseando a Crenshaw (1991: 1242), tal "interseccionalidad" se refiere a que las experiencias de las parteras tradicionales revelan cómo diferentes estructuras se intersectan e interrelacionan, demostrando cómo la clase social no es independiente de la etnia o del género y que su interrelación crea situaciones de discriminación y desigualdad social que son complejas de explicar, de resolver y de que se tomen en cuenta.

${ }^{18} \mathrm{Al}$ respecto ver Ransom y Yinger (2002), OPS (1998), y Begum y Kabir (1990), citado en O’Rourke (1995).

\section{Referencias bibliográficas}

Araujo, Galba et al., (1984) "Parteras tradicionales en la atención obstétrica del noreste de Brasil” en Bol Of Sanit Panam. Vol. 96, núm. 2, pp. 147-159.

Birn, Anne-Emanuelle, (2006) Marriage of Convenience: Rockefeller International Health and Revolutionary Mexico. Nueva York, University of Rochester Press.

Carrillo, Ana María, (1998) "Profesiones sanitarias y lucha de poderes en el México del siglo XIX" en Asclepio, L-2, pp. 149-168.

Colom, Artur, (2003) "iEl retorno de los elefantes blancos? África, deuda, y pobreza” en Revista de Economía Crítica. Núm. 1, pp. 175-187.

Crenshaw, Kimberlé, (1991) "Mapping the Margins: Intersectionality, Identity Politics, and Violence against Women of Color" en Stanford Law Review. Vol. 43, núm. 6, pp. 1241-1299.

Ehrenreich, Bárbara y Deirdre English, (1988 [1981]) Brujas, comadronas y enfermeras. Historia de las sanadoras. Dolencias y trastornos. Política sexual de la enfermedad. Barcelona, La Sal Edicions de las Dones.

Fondo de las Naciones Unidas para la Infancia (UNICEF), (2009) Estado mundial de la infancia 2009. Salud materna y neonatal. Nueva York, UNICEF. Disponible en: http://www.unicef.org/spanish/publications/ files/SOWC_2009_Main_Report_LoRes_PDF_SP_ USLetter_03112009.pdf [consultado el 5 de enero de 2012].

Goodburn, Elizabeht et al., (2000) "Training Traditional Birth Attendants in Clean Delivery Does not Prevent Postpartum Infection" en Health Policy and Planning. Vol. 15, núm. 4, pp. 394-399. Disponible en: http:// www.unicef.org/spanish/specialsession/about/ sgreport-pdf/09_MaternalMortality_D734lInsert Spanish.pdf [consultado el 15 de junio de 2008].

Mangay-Manglacas, A. y H. Pizurki (eds.), (1981) La partera tradicional en siete países. Estrategias para su adiestramiento. Ginebra, Organización Mundial de la Salud.

Martínez, Alvar y José Pardo, (2001) "Un conflicto profesional, un conflicto moral y un conflicto de género: los debates en torno a la atención al parto en 
tiempos de Josefa Amar y Borbón" en Cronos. Núm. 4 pp. 3-27.

Naciones Unidas. Centro de Información México, Cuba y República Dominicana, (s.f.) "Países menos adelantados" [en línea]. Disponible en: http:/ www.cinu.org.mx/temas/desarrollo/dessocial/ldc/ paisesmadel.htm [consultado el 18 de junio de 2008].

Naciones Unidas, (1995) Informe de la Conferencia Internacional sobre la Población y el Desarrollo. Nueva York, Naciones Unidas.

Naciones Unidas, (1999) Conferencia internacional sobre la población y el desarrollo $(\mathrm{ICPD}+5)$ [en línea]. Disponible en: http://www.un.org/es/development/devagenda/ population.shtml [consultado el 19 de noviembre 2013].

Naciones Unidas, (2000) Objetivos de desarrollo del milenio [en línea]. Disponible en: http://www.unicef.org/ spanish/mdg/ [consultado el 15 de junio 2008].

OMS. Organización Mundial de la Salud, (1966) Comité de expertos de la OMS sobre la función de la partera en la asistencia a la madre. Informe de un comité de Expertos de la OMS. Ginebra, Organización Mundial de la Salud. Disponible en: http://whqlibdoc.who.int/trs/WHO TRS_331_spa.pdf [consultado el 18 de noviembre de 2013].

OMS. Organización Mundial de la Salud, (2005) Informe sobre la salud en el mundo 2005. Ginebra, OMS.

OMS. Organización Mundial de la Salud, (2008) Mortalidad materna en 2005: estimaciones elaboradas por la OMS, el UNICEF, el UNFPA y el Banco Mundial. S.l., OMS. Disponible en: http://whqlibdoc.who. int/publications/2008/9789243596211_spa.pdf [consultado el 18 de noviembre de 2013].

OPS.OrganizaciónPanamericanadelaSalud,(1998)“25.a Conferencia Sanitaria Panamericana. Documentos de trabajo". Washington, 21 al 25 de septiembre de 1998. Disponible en: http://www.paho.org/hq/index. php?option=com_content $\&$ view $=$ article $\&$ id $=2807$ $\% 3 \mathrm{~A} 25$ th-pan-american-sanitary-conference\%2C21-25-sept. $-1998 \&$ catid $=2113 \% 3$ Agbo-csp $25 \% 2 C$ 21-25-sept-1998\& \&lang=es [consultado el 17 de noviembre de 2013].

OPS. Organización Panamericana de la Salud, (2002) “26.a Conferencia Sanitaria Panamericana. 54.a sesión del comité regional. Documentos de trabajo". Washington, 23 al 27 de septiembre de 2002. Disponible en: http:// wwwl.paho.org/spanish/gov/csp/csp26index-s.htm [consultado el 17 de octubre de 2013].

OPS. Organización Panamericana de la Salud y OMS. Organización Mundial de la Salud, (2002) Promoción de la medicina y terapias indígenas en la atención primaria de salud: el caso de los garifunas de Honduras. Disponible en: http://www.paho.org/Spanish/AD/THS/OS/indigserie-17.pdf [consultado el 18 de junio de 2008]

O’Rourke, Kathleen, (1995) “Evaluación de un programa de capacitación de parteras tradicionales en Quetzaltenango, Guatemala" en Bol of Sanit Panam. Vol. 119, núm. 6, pp. 503-514.

Oxfam, (2005) Más allá de la iniciativa HIPC. Reino Unido, Oxfam.

Piferrer, Tomás (impr.), (1765) Compendio en el arte de partear: compuesto para el uso de los reales colegios de cirugía. Con licencia. Barcelona, Tomás Piferrer. Disponible en: http://babel.hathitrust.org/cgi/pt?id= ucm.5329l15362; view=lup;seq=5 [consultado el 15 de octubre de 2013].

Ransom, Elisabeth y Nancy Yinger, (2002) Por una maternidad sin riesgos. Cómo superar los obstáculos en la atención a la salud materna. Washington, Population Reference Bureau.

Reich, Warren Thomas (ed.), (1995) Encyclopedia of Bioethics, Revisededition. Nueva York, Simon \& Schuster Macmillan, pp. 1340-1342, 1543-1549 y 2577-2584.

Rodríguez, Marta et al., (2012) "Medicalización en las mujeres embarazadas y parturientas: Una aproximación desde terapia ocupacional parte I" en TOG. Vol. 9, núm. 15, pp. 1-18.

Towler, Jean y Joan Bramall, (1997) Comadronas en la historia y en sociedad. Barcelona, Masson.

UNFPA. Fondo de Población de las Naciones Unidas, (1996) Estado de la población mundial 1996: población, desarrollo y el futuro urbano. Nueva York, Fondo de Población de las Naciones Unidas.

UNFPA. Fondo de Población de las Naciones Unidas, (2002a) Estado de la población mundial 2002: población, pobreza y oportunidades. Nueva York, Fondo de Población de las Naciones Unidas. 
UNFPA. Fondo de Población de las Naciones Unidas, (2002b) Informe anual 2002. Nueva York, Fondo de Población de las Naciones Unidas.

UNFPA. Fondo de Población de las Naciones Unidas (2002c), Mortalidad materna. Actualización 2002. Nueva York, Fondo de Población de las Naciones Unidas.

UNFPA. Fondo de Población de las Naciones Unidas (2002d), Para entender las causas de la mortalidad materna. Nueva York, UNFPA.

UNFPA. Fondo de Población de las Naciones Unidas, (2004a) Estado de la población mundial 2004. El Conseso de El Cairo, diez años después: población, salud reproductiva y acciones mundiales para eliminar la pobreza. Nueva York, Fondo de Población de las Naciones Unidas.

UNFPA. Fondo de Población de las Naciones Unidas, (2004b) Mortalidad materna-actualización 2004. El parto en buenas manos. Nueva York, Fondo de Población de las Naciones Unidas.

UNFPA. Fondo de Población de las Naciones Unidas, (2008) Estado de la población mundial 2008. Ámbitos de convergencia: cultura, género y derechos humanos. Nueva York, Fondo de Población de las Naciones Unidas.
UNFPA. Fondo de Población de las Naciones Unidas, (2009) Informe anual 2009. Nueva York, Fondo de Población de las Naciones Unidas.

UNFPA. Fondo de Población de las Naciones Unidas, (201la) Estado de la población mundial 2011.7 mil millones de personas. Su mundo, sus posibilidades. Nueva York, Fondo de Población de las Naciones Unidas.

UNFPA. Fondo de Población de las Naciones Unidas, (201lb) El estado de las parteras en el mundo 2011. Nueva York, Fondo de Población de las Naciones Unidas.

UNFPA. Fondo de Población de las Naciones Unidas, (201lc) Informe anual 2011. Nueva York, Fondo de Población de las Naciones Unidas.

UNFPA. Fondo de Población de las Naciones Unidas, (2012) Ofrecer esperanza y salvar vidas-Inversiones en partería. Nueva York, Fondo de Población de las Naciones Unidas. Disponible en: http://www.unfpa. org/webdav/site/global/shared/factsheets/srh/SPSRH\%20fact\%20sheet-Midwifery.pdf [consultado el 25 de noviembre de 2013].

Usandizaga, Manuel, (1944) Historia de la obstetricia y la ginecología en España. Santander, Aldus. 